\title{
Aplicação do SNC-AP nas entidades do Serviço Nacional de Saúde
}

\section{The application of the SNC-AP in the National Health Service}

\author{
Carlos Nunes \\ Escola Superior de Gestão, Hotelaria e Turismo, Universidade do Algarve, Portugal \\ cmnunes@ualg.pt \\ Joaquim Sant'Ana Fernandes \\ Escola Superior de Gestão, Hotelaria e Turismo, Universidade do Algarve, Faro, Portugal \\ jsfer@ualg.pt

\section{Cristina Gonçalves} \\ Escola Superior de Gestão, Hotelaria e Turismo, Universidade do Algarve, Faro, Portugal \\ cjesus@ualg.pt
}

\begin{abstract}
Resumo
A partir de 1 de janeiro de 2018 todos os serviços e organismos da administração central, regional e local, que não tenham natureza, forma e designação de empresa pública bem como o subsetor da segurança social e as entidades públicas reclassificadas, tais como entidades do Serviço Nacional de Saúde, passam a aplicar o Sistema de Normalização Contabilística - Administração Pública (SNC-AP). Auscultaram-se os responsáveis pela contabilidade de 36 entidades do Serviço Nacional de Saúde sobre a mudança de referencial e as respetivas implicações. Em média, estes valorizam positivamente o SNC-AP, contudo atribuem maior importância ao Plano Oficial de Contabilidade Ministério da Saúde. Estes profissionais reconhecem dificuldades técnicas na transição e impactos negativos nos ativos e passivos, mas positivos nos capitais próprios.
\end{abstract}

Palavras-Chave: perceção dos preparadores; POC-MS; SNC-AP; transição.

\begin{abstract}
From the 1st January 2018 onwards, all the central, regional and local administration services and organisations that do not have the nature, form and designation of a public company, as well as the social security sector and the reclassified public entities, such as the National Health Service, will start to apply the Sistema de Normalização Contabilística - Administração Pública (SNC-AP). The people in charge of accounting for 36 National Health Service entities were interviewed about this change and its implications. On average, the SNC-AP was positively valued, although the POC-MS was considered of more importance. The technical difficulties in this transition are recognized along with the negative impacts on assets and liabilities and the positive impacts on the owner's equity.
\end{abstract}

Keywords: preparers' perception; POC-MS; SNC-AP; transition.

\section{Introdução}

Até início da década de 90, os objetivos centrais dos sistemas contabilísticos das entidades públicas relacionavam-se com o controlo da execução orçamental e com o controlo da legalidade das operações, sendo que as obrigações e os direitos eram reconhecidos quando se verificava a entrada e saída de valores monetários. Neste contexto, a unigrafia ou regime

Dos Algarves: A Multidisciplinary e-Journal, 29 - 2017.

ISBN 2182-5580 ๑ ESGHT-University of the Algarve, Portugal.

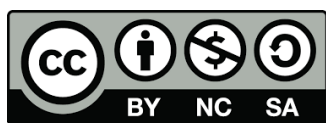

To cite this article: C. Nunes, J. S.Fernandes e C. Gonçalves (2017). Aplicação do SNC-AP nas entidades do Serviço Nacional de Saúde. Dos Algarves: A Multidisciplinary e-Journal, 29, 49-63. doi: 10.18089/DAMeJ.2017.29.3 
do caixa, era o modelo de registo contabilístico predominante na administração pública portuguesa.

O ponto de viragem ocorreu em 1992 com a designada Reforma da Administração Financeira do Estado e com a implementação do Plano Oficial de Contabilidade Pública (POCP) em 1997. Deste modo, foram estabelecidas as condições para uma alteração de um paradigma - de eminentemente orçamental para a prestação de informação orientada para a tomada de decisão.

Volvidos que foram sensivelmente vinte anos após a entrada em vigor do POCP, a sua alteração era expectável e de facto ocorreu com a aprovação do Sistema de Normalização Contabilística (SNC) para as Administrações Públicas (AP) em setembro de 2015, aproximando-o do SNC empresarial e das International Public Sector Accounting Standards (IPSAS) emitidas pelo International Public Sector Accounting Standards Board. Com a entrada em vigor do SNC-AP a partir de 1 de janeiro de 2018, o POCP e os respetivos planos setoriais serão revogados.

Deste modo, o setor público administrativo (SPA) - serviços não autónomos da administração central, regional e local, a segurança social e as entidades reclassificadas na administração pública, incluindo, desta forma, as entidades do Serviço Nacional de Saúde (SNS) - passará a reconhecer e a mensurar as suas operações e outros acontecimentos de acordo com o referencial SNC-AP. As demonstrações financeiras (DF) do SNS são preparadas pelos respetivos responsáveis pela contabilidade, que podem possuir, ou não, certificação profissional.

Este trabalho suporta-se no estudo de Nunes, Fernandes e Gonçalves (2016) aplicado às unidades hospitalares, nas quais a responsabilidade pela preparação das DF é assegurada por um contabilista certificado, agora ampliado às restantes entidades do SNS, independentemente da qualificação profissional do responsável pela contabilidade.

O estudo pretende contribuir para providenciar evidência empírica sobre os efeitos da adoção do SNC-AP e antecipar o efeito da transição, considerando uma amostra mais alargada.

No que se refere à metodologia utilizada, recorreu-se ao questionário de Nunes et al. (2016), assegurando-se deste modo a comparabilidade dos resultados, disponibilizado online. Conclui-se, em termos gerais, que não existem diferenças significativas na valorização atribuída a ambos os normativos, exceto entre os profissionais com vinte ou mais anos de serviço, que preferem o Plano Oficial de Contabilidade - Ministério da Saúde (POC-MS), em detrimento do SNC-AP, e que também são descrentes em relação à utilidade das DF preparadas no âmbito deste normativo.

Para além desta primeira parte, o presente artigo é constituído por mais quatro. Assim, numa segunda parte, enquadra-se o tema e formulam-se as hipóteses de investigação e, numa terceira descreve-se o estudo empírico. Os resultados e a respetiva análise são apresentados na quarta parte, seguindo-se uma quinta, e última secção, com as principais conclusões e limitações da investigação.

\section{Enquadramento do tema e definição das hipóteses}

Conforme referem Nogueira e Carvalho (2006), o setor público português, com a revolução do 25 de Abril de 1974, foi confrontado com profundas alterações relacionadas com a passagem de um regime político ditatorial para a democracia. A consequente separação de 
poderes, legislativo e executivo, bem como a independência dos tribunais, entre os quais o Tribunal de Contas, conduziu a diversas modificações no regime legal, no que diz respeito à contabilidade pública. Outro marco relevante para a contabilidade pública ocorreu em 1986, com a adesão de Portugal à antiga Comunidade Económica e Europeia, quando foi expectável "uma reforma da contabilidade pública portuguesa semelhante à dos países mais desenvolvidos" (Nogueira \& Carvalho, 2006: 2). Todavia, o que se verificou foi somente uma alteração ao Plano Oficial de Contabilidade (POC), em 1989, decorrente da IV Diretiva da Comunidade Europeia, sendo esse diploma considerado um instrumento determinante para o desenvolvimento da contabilidade em Portugal (vide Pires, 2009). No entanto, apenas a partir dos anos 90, com a aprovação da Lei n. ${ }^{\circ} 8 / 1990$, de 20 de fevereiro (Lei de Bases da Contabilidade Pública), se iniciou o processo de reforma da Administração Pública, cujo objetivo consistia na aproximação ao sistema contabilístico utilizado pelas empresas privadas (Nogueira \& Carvalho, 2006). Esse novo sistema de contabilidade pública, designado por POCP, foi aprovado pelo Decreto-Lei n. ${ }^{\circ} 232 / 1997$, de 3 de setembro.

Na realidade, o POCP foi um marco histórico para a contabilidade do setor público, uma vez que a sua publicação se enquadra, por um lado, na reforma da administração financeira do Estado e, por outro, "constitui um instrumento indispensável para dotar o Estado de um sistema de contas adequado às necessidades de uma Administração Pública moderna" (POCP, ponto 1 - Introdução). Com a aprovação do POCP, foi criada, no âmbito do Ministério das Finanças, a Comissão de Normalização Contabilística da Administração Pública (CNCAP), cuja missão era assegurar a normalização e acompanhar a aplicação e o aperfeiçoamento do POCP e dos planos setoriais públicos, de uma forma gradual, garantindo a necessária segurança e eficácia (artigo $4 .^{\circ}$, Decreto-Lei n. ${ }^{\circ}$ 232/1997). Refira-se que todos estes planos tiveram por base o POC, normativo que, entretanto, foi substituído pelo SNC. A CNCAP foi extinta em 2011 e as suas atribuições foram integradas na Comissão de Normalização Contabilística. A mencionada CNCAP já referenciava a necessidade de um "novo Sistema Público de Normalização Contabilística", no âmbito de um conjunto de orientações genéricas relativo à consolidação de contas no setor público administrativo, o que se concretizou com a aprovação do SNC-AP.

Neste contexto, Nunes et al. (2016) realizaram um estudo da opinião dos contabilistas certificados das unidades hospitalares sobre a aceitação do novo normativo, tendo concluído pela sua preferência em relação ao POC-MS.

$\mathrm{Na}$ continuação desse estudo, o presente trabalho, no qual se inclui uma amostra mais ampla, inserindo também os contabilistas sem certificação profissional, define-se como hipótese, formulada pela positiva:

H1: Os responsáveis da contabilidade das entidades do SNS consideram que o SNC-AP é mais adequado que o POC-MS.

Diversos estudos procuram antecipar a aplicação de um novo sistema contabilístico no setor público. Muitos trabalhos analisam, de forma comparativa, o POCP e as IPSAS (ou, em alguns estudos, o POCP e o SNC), ressalvando as suas virtualidades. Refira-se Cruz (2012), que encontra pontos fortes no POCP, designadamente por apresentar virtualidades relevantes relativamente ao normativo internacional, uma vez que preconiza instrumentos de controlo orçamental mais detalhados e mais adequados ao nível do desenvolvimento de práticas contabilísticas orçamentais. Por sua vez, as IPSAS destacam-se na vertente 
financeira/patrimonial na qual existem significativas áreas de aperfeiçoamento do normativo português. Gonçalves (2011) conclui que existe uma perceção da necessidade de uma adaptação do POCP ao SNC para incrementar a accountability. Nesta linha de conclusões, Pinho (2014) refere que a adoção das IPSAS produz um impacto no relato financeiro, quer ao nível do conjunto completo de DF obrigatórias, quer na sua estrutura e conteúdo. Também Barbosa (2009), com base num inquérito realizado no seio das forças aéreas brasileiras e portuguesas, analisou as vantagens e as desvantagens da harmonização contabilística das IPSAS na contabilidade pública destes dois países, concluindo pela relevância que a padronização das DF tem em ambos os territórios como parâmetro de comparabilidade.

No sentido de aferir a importância atribuída às DF preparadas no âmbito do SNC-AP define-se como hipótese formulada pela positiva:

H2: Os responsáveis da contabilidade das entidades do SNS consideram que o

SNC-AP permite a apresentação de DF mais comparáveis entre as diferentes entidades do setor administrativo do Estado.

O Decreto-Lei de aprovação do SNC-AP previa a criação de um regime simplificado de contabilidade pública para as entidades de menor dimensão, em diploma a definir, assim como a elaboração de um Manual de Implementação no qual seriam estabelecidas instruções para a respetiva adoção, pela primeira vez, em sede do diploma de aprovação do novo normativo, já que as entidades públicas sujeitas ao SNC-AP terão de preparar o balanço de abertura relativo ao exercício de 2017 de acordo com o novo normativo. Assim, este processo implica um conjunto de ajustamentos ao último balanço preparado de acordo com os anteriores normativos de contabilidade pública. À data, estão aprovados o Manual de Implementação do SNC-AP e o Regime Simplificado do SNC-AP, instrumentos previstos no citado Decreto-Lei.

Em virtude dos ajustamentos mencionados anteriormente, o SNC-AP, no seu artigo $14 .^{\circ}$, n. ${ }^{\circ}$ s 3 e 4, estabelece que os ajustamentos resultantes da mudança das políticas contabilísticas que se verifiquem, na adoção do SNC-AP, devem ser reconhecidos no saldo de resultados transitados no período em que os itens são reconhecidos e mensurados. Estabele também que as entidades públicas devem considerar ainda os correspondentes ajustamentos no período comparativo anterior. Neste sentido, a Norma de Contabilidade Pública 1 contém um conjunto de divulgações que deve ser efetuado no ano de transição, isto é, no primeiro período de relato em que a entidade aplica pela primeira vez o SNC-AP. Alguns autores, como Rodrigues (2015), debruçam-se sobre a análise dos impactos em algumas rúbricas. Este autor analisa especialmente as consequências de uma revalorização dos bens de domínio público, enquanto Silva e Carvalho (2007) confrontam o reconhecimento das provisões, dos passivos contingentes e dos ativos contingentes nos dois normativos (POCP e IPSAS 19). Nesta linha de investigação, Correia e Gonçalves (2010) comparam o reconhecimento dos ativos fixos tangíveis na IPSAS 17 e na Norma Internacional de Contabilidade 16, enquanto Coelho (2014) destaca as diferenças em diversos aspetos entre 0 POC-Educação e as IPSAS correspondentes. 
Assim, define-se como hipótese, formulada pela positiva:

H3: Os responsáveis da contabilidade das entidades do SNS consideram que a implementação do SNC-AP conduz a impactos significativos no Balanço das entidades que integram o SNS.

A implementação de um sistema contabilístico na administração pública assente nos conceitos das normas internacionais para o setor público administrativo deu origem a diversos estudos e artigos de opinião que, em certo modo, procuram fazer análises comparativas, antecipar as vantagens, os impactos e os condicionalismos associados a essa implementação. Deste modo, refira-se o levantamento bibliográfico realizado por França e Jesus (2014). Estes investigadores analisaram diversos contributos de âmbito nacional, que, no seu conjunto, permitem ter uma visão de alguns dos diversos estudos que este tema suscitou.

Parece estar instalada a ideia, a priori, de que as normas internacionais constituem a melhor prática a ser seguida para as contas públicas (Jorge, 2012a, 2012b), sem que, contudo, existam estudos empíricos sobre as consequências desta adoção global, ou seja, ainda não estão avaliados os resultados das reformas passadas e em curso relacionadas com o POCP. Nesta linha de preocupações, Santos e Pinho (2014) referem que é possível concluir que a implementação em Portugal das IPSAS parece inevitável, no entanto, existem poucas evidências sobre se a harmonização das normas contabilísticas nacionais com base nas IPSAS resultará na efetiva harmonização das práticas contabilísticas. Também Ferreira (2013) enfatiza o processo inacabado de implementação do POCP e, em simultâneo, chama a atenção para a necessidade de preparar a adoção de um normativo baseado nas IPSAS, para atenuar a eventual impreparação dos recursos humanos, técnicos e organizacionais. Outra linha de estudos preocupa-se com os utilizadores e os preparadores da informação. Gomes, Fernandes e Carvalho (2015) concluíram existir um elevado nível de coesão entre os diferentes stakeholders sobre os estímulos da reforma e o seu conteúdo. Já Nogueira e Carvalho (2006) preocuparam-se em conhecer a opinião de diversos especialistas (no âmbito do POCP), designadamente docentes do ensino superior e técnicos. O seu estudo releva uma implementação insatisfatória dos planos de contabilidade pública devido, nomeadamente, à falta de meios humanos com qualificações adequadas, às dificuldades na interpretação de conceitos contabilísticos por parte do pessoal técnico e à insuficiência de recursos tecnológicos. Destaca-se ainda a elevada importância atribuída a uma estrutura concetual para a contabilidade pública. Neste sentido, formula-se como hipótese de partida, expressa pela positiva:

H4: Os responsáveis pela contabilidade das entidades do SNS estão preparados para a mudança de referencial contabilístico.

O presente estudo insere-se na linha de investigação desenvolvida por Gomes et al. (2015), Nogueira e Carvalho (2006) e Nunes et al. (2016) na medida em que pretendem auscultar os recursos humanos diretamente envolvidos na implementação do novo sistema contabilístico. 


\section{Estudo empírico}

\subsection{Problema e objetivos da investigação}

Recorde-se que o Decreto-Lei n. ${ }^{\circ}$ 192/2015, de 11 de setembro, aprovou o SNC-AP, que entra em vigor a partir de 1 de janeiro de 2018 e cujo objetivo é garantir a harmonização do quadro contabilístico nacional com as normas internacionais. Importa, desde logo, salientar que a transposição para o novo normativo não se limita a um elenco de alterações concretas relativas à forma de registo e relato de transações económicas, mas sim, e principalmente, a uma mudança de filosofia de base no reporte de informação financeira. Os responsáveis pela contabilidade do SNS serão chamados a gerir esse processo de mudança, quer ao nível da sua preparação técnica, quer na orientação dos procedimentos associados à implementação das novas regras contabilísticas.

Assim sendo, pretende-se, com este estudo, aferir se existem diferenças estatisticamente significativas entre as opiniões dos responsáveis pela preparação das DF do SNS, quanto:

(1) à adequação do POC-MS versus SNC-AP às finalidades de relato financeiro;

(2) à maior adequabilidade das DF preparadas no âmbito do SNC-AP;

(3) às alterações previsíveis nos principais agregados do balanço;

(4) ao domínio técnico do SNC-AP.

\subsection{Universo e amostra}

Atendendo aos objetivos do estudo, foram consideradas as entidades do SNS, cujo universo é composto por cinquenta e três entidades (Apêndice A). Das cinquenta e três entidades, trinta e nove são do Setor Empresarial do Estado e catorze do Setor Público Administrativo. Estas cinquenta e três entidades correspondem a: vinte e três Centros Hospitalares, nove Hospitais, seis Institutos, oito Unidades Locais de Saúde, cinco Administrações Regionais de Saúde, um Centro de Reabilitação e à Administração Central do Sistema de Saúde (Apêndice A).

A amostra é constituída pelos respondentes ao questionário, que totalizou 36 responsáveis pela contabilidade de entidades do SNS, correspondendo a 68\% do universo, maioritariamente do género masculino (61\%) e com idades compreendidas entre os 40 e os 50 anos (58\%). Em relação às habilitações literárias todos os inquiridos têm formação superior, na sua grande maioria (83\%) formação ao nível do primeiro ciclo (licenciatura) e 17\% apresentam habilitações ao nível do segundo ciclo do ensino superior (mestrado). Cerca de 17\% dos inquiridos têm 20 ou mais anos de tempo de serviço na entidade. Cerca de $86 \%$ da amostra são contabilistas certificados.

\subsection{Instrumento de recolha de dados}

O instrumento de recolha de dados depende do fenómeno a investigar (Ryan, Scapens \& Theobald, 2002). Considerando que se pretende aferir a opinião dos responsáveis pela contabilidade do SNS, relativamente aos aspetos já referidos, entendeu-se que o instrumento mais adequado era o inquérito por questionário disponibilizado numa ferramenta do Gmail (Google), não sendo necessária a presença de um entrevistador (Marconi \& Lakatos, 1999). 
Utilizou-se o questionário de Nunes el al. (2016), para assegurar comparabilidade dos resultados.

O questionário é constituído por uma série ordenada de questões e escalas. Foram utilizadas questões fechadas relativamente a variáveis biográficas e instrumentos que operacionalizam as variáveis em estudo, com base em itens sob a forma de escala crescente tipo Likert (de 1 a 7 ).

O questionário é composto por 6 questões de caracterização e 28 itens de opinião sobre diversos aspetos, quer do normativo ainda em vigor (POC-MS), quer sobre a aplicação do SNC-AP. Os objetivos de análise são (1) a adequação do POC-MS versus SNC-AP para as finalidades de reconhecimento e relato financeiro (oito itens); (2) a qualidade das DF produzidas no âmbito do SNC-AP (seis itens); (3) o domínio técnico do SNC-AP (quatro itens) e (4) as alterações previsíveis nos principais agregados do balanço (dez itens).

No sentido de minimizar os riscos subjacentes a este instrumento de recolha de dados, mais precisamente uma incorreta interpretação das questões e a não resposta, na introdução do questionário, explicitaram-se os objetivos da investigação e garantiu-se a confidencialidade dos dados.

Para medir a consistência interna das escalas, utilizou-se o teste de Alfa de Cronbach cujos a oscilam entre os 0,852 e os 0,948 pelo que se deduz que o mesmo apresenta uma boa consistência interna.

Tabela 1. Alfa de Cronbach

\begin{tabular}{|c|c|c|}
\hline Fator & N. $^{\circ}$ itens & Alfa de Cronbach \\
\hline POC-MS & 4 & 0,869 \\
\hline SNC-AP & 4 & 0,948 \\
\hline DF SNC AP & 6 & 0,928 \\
\hline Impacto SNC AP & 5 & 0,852 \\
\hline
\end{tabular}

Para testar as suposições sobre a população, conjeturadas nos objetivos do estudo, foram utilizadas estatísticas descritivas e testes não paramétricos e testes paramétricos para a diferença de médias entre os fatores.

\section{Resultados e análise}

Para o teste de igualdade das distribuições entre os grupos de variáveis independentes para cada uma das variáveis dependentes, utilizou-se o teste de Mann-Whitney no caso de 2 amostras independentes e o teste de Kruskal Wallis para o caso de 3 ou mais amostras independentes. Considerou-se o nível de significância inferior a 0,05 ( $p$-value <0,05). A intensidade da relação entre variáveis foi analisada através de coeficiente Ró de Spearman. A análise tomou em consideração as características da amostra (género, idade, habilitações (licenciados e pós-graduados), anos de experiência (menos de 20 anos e 20 ou mais anos na função), certificação profissional (contabilista certificado ou não) e a dimensão das entidades (proxy volume de negócios por trabalhador - superior e inferior ao valor médio) como variáveis explicativas das diferenças de opinião.

(1) Adequação o POC-MS versus SNC-AP. 
Recolheu-se a opinião sobre a adequabilidade do atual normativo e do SNC-AP, cuja utilidade é ainda desconhecida por não estar em aplicação. As respostas mostram existir uma opinião favorável sobre o POC-MS ( $\overline{\mathrm{x}}=4,611 ; \sigma=1,351)$, com uma maior valorização na sua função relacionada com a forma legal das operações $(\overline{\mathrm{x}}=4,778 ; \sigma=1,436)$ e uma menor valorização da comparabilidade das DF ( $\overline{\mathrm{x}}=4,472 ; \sigma=1,715)$.

A adequabilidade do SNC-AP apresenta uma média ligeiramente menor e desvio padrão superior $(\overline{\mathrm{X}}=4,534, \sigma=1,448)$, sendo que o item mais valorizado é a adequação da informação às necessidades dos utilizadores públicos $(\overline{\mathrm{x}}=4,556 ; \sigma=1,462)$.

Não se confirma, em termos gerais, haver diferença significativa entre a valorização atribuída ao POC-MS e SNC-AP. Contudo, numa análise em função das diversas variáveis consideradas, constata-se uma diferença significativa na opinião entre os responsáveis, em função da sua antiguidade. Enquanto no conjunto dos colaboradores com menos de 20 anos de tempo de serviço não existe diferença significativa no valor que atribuem a ambos os normativos, já os que se encontram no escalão dos 20 ou mais anos de tempo de serviço atribuem claramente mais importância ao POC-MS ( $t=2.2409 ; \mathrm{p}=0.0379)$.

Tabela 2. SNC-AP e escalões de antiguidade

\begin{tabular}{|l|c|c|c|c|c|}
\hline Tempo de serviço & $\mathrm{N}$ & POC-MS & SNC-AP & $t$ & P-value \\
\hline $0-20$ anos & 26 & 4,36 & 4,84 & -1.3626 & 0.1791 \\
\hline Mais de 20 & 10 & 5.25 & 3.72 & 2.2409 & 0.0379 \\
\hline
\end{tabular}

Também o género contribui para diferentes opiniões quanto à capacidade do SNC-AP atender à forma legal das operações. O nível de concordância é superior nos homens $(4,9)$ para uma média de 4 nas mulheres $(p=0,083)$. Por outro lado, verifica-se uma correlação negativa significativa ( $p=0,081$ ) de 0,294 (fraca) entre as opiniões sobre o POC-MS e o SNC$\mathrm{AP}$, revelando, que em parte, estas opções não são alternativas.

As variáveis certificação profissional, habilitações e dimensão das entidades, não apresentaram qualquer significado para a explicação dos resultados.

(2) Qualidade das demonstrações financeiras elaboradas segundo o SNC-AP.

Recorreu-se a vários atributos para medir a qualidade da informação no âmbito do SNCAP, no qual se verifica uma valorização positiva $(\bar{x}=4,75, \sigma=1,307)$. O facto de o SNC-AP ser mais exigente na quantidade e qualidade da informação é o atributo mais valorizado $(\overline{\mathrm{X}}=5$, $728 ; \sigma=1,596)$, por oposição à forma legal e à comparabilidade, atributos menos valorizados $(\overline{\mathrm{x}}=4,556)$.

Contata-se uma forte correlação positiva entre a qualidade das DF e a opção pelo SNCAP $(\rho=0,930 ; p<0.001)$, o que reforça a coerência das respostas (a correlação entre a qualidade e o POC-MS é negativa, mas não significativa estatisticamente).

Neste fator encontra-se um posicionamento diferente entre os profissionais com mais de 20 anos de tempo de serviço, que de forma coerente com os resultados anteriores, encontram menos vantagens na utilização do SNC para preparação das demonstrações financeiras. Estas diferenças estatisticamente significativas (para $p<0,1$ ) estendem-se a um 
conjunto de questões, nas quais os profissionais com mais tempo de serviço apresentam sistematicamente menor valorização.

Tabela 3. Qualidade das DF e escalóes de antiguidade

\begin{tabular}{|l|c|c|c|c|c|}
\hline Tempo de serviço & SNC DF & $\begin{array}{c}\text { Qualidade e } \\
\text { quantidade } \\
\text { informação }\end{array}$ & $\begin{array}{c}\text { Atende à } \\
\text { substância } \\
\text { das operações }\end{array}$ & $\begin{array}{c}\text { Atende à } \\
\text { forma legal }\end{array}$ & $\begin{array}{c}\text { Atende às } \\
\text { necessidades } \\
\text { dos utilizadores }\end{array}$ \\
\hline $\begin{array}{l}\text { Menos de 20 anos } \\
\text { 20 ou mais anos }\end{array}$ & 5,06 & 5,7 & 5,0 & 4,9 & 5,0 \\
& 4,93 & 0,8 & 3,6 & 3,8 \\
\hline $\begin{array}{c}\text { U Mann Whitney } \\
\text { (p-value) }\end{array}$ & 0,041 & 0,068 & 0,063 & 0,031 & 0,049 \\
\hline
\end{tabular}

O género também parece influenciar a perceção sobre a maior exigência do SNC-AP quanto à quantidade e qualidade de informação $(p=0,061)$. Enquanto na população masculina apresenta uma média de 5,6, a média da população feminina é de apenas 4,7, interpretando-se haver uma menor valorização deste atributo por parte destas. Note-se que a apreciação dos homens $(\overline{\mathrm{x}}=4,95)$ sobre as DF preparadas no âmbito do SNC-AP também é superior à apreciação feita pelas mulheres $(\overline{\mathrm{X}}=4,44)$, embora neste aspeto a diferença não seja estatisticamente significativa.

À semelhança da hipótese anterior, as variáveis certificação profissional, habilitações e dimensão das entidades, não apresentaram qualquer significado para a explicação dos resultados.

(3) Alterações previsíveis nos principais agregados do balanço.

Quanto ao previsível impacto que a adoção do SNC-AP terá no balanço, a expetativa é de impactos moderados $(\overline{\mathrm{X}}=4,194 ; \sigma=1,508)$, sendo expectável que o maior impacto se verifique nos ativos não correntes $(\overline{\mathrm{x}}=4,806)$ e o menor no passivo não corrente $(\overline{\mathrm{x}}=3,667)$.

Quanto à natureza dos impactos, a maioria dos inquiridos antevê efeitos negativos nos ativos (correntes e não correntes) e uma diminuição dos passivos não correntes. O efeito combinado esperado é de acréscimo dos capitais próprios (64\%). Refira-se que as respostas de impacto negativo (diminuições de ativo e aumento de passivo) representam $58 \%$ das opiniões, contudo a expetativa maioritária é de um aumento dos capitais próprios.

Tabela 4. Alterações positivas e negativas nas rubricas do balanço

\begin{tabular}{|l|c|c|c|c|c|c|c|c|c|c|}
\hline $\begin{array}{c}\text { Impacto do } \\
\text { SNC-AP }\end{array}$ & \multicolumn{2}{|c|}{$\begin{array}{c}\text { Ativo não } \\
\text { corrente }\end{array}$} & \multicolumn{2}{|c|}{ Ativo corrente } & \multicolumn{2}{c|}{$\begin{array}{c}\text { Passivo não } \\
\text { corrente }\end{array}$} & \multicolumn{2}{c|}{ Passivo corrente } & \multicolumn{2}{c|}{ Capital próprio } \\
\hline Aumento & 10 & $28 \%$ & 10 & $28 \%$ & 14 & $39 \%$ & 18 & $50 \%$ & 23 & $64 \%$ \\
\hline Diminuição & 26 & $72 \%$ & 26 & $72 \%$ & 22 & $61 \%$ & 18 & $50 \%$ & 13 & $36 \%$ \\
\hline
\end{tabular}

Verificam-se diferenças significativas sobre o impacto das alterações induzidas pelo SNC AP, quer em função da dimensão da entidade, quer quanto ao tempo de serviço dos profissionais. 
Tabela 5. Alterações nas rubricas do balanço (dimensão e tempo de serviço)

\begin{tabular}{|c|c|c|c|c|c|c|c|}
\hline \multicolumn{2}{|c|}{ Variáveis } & $\begin{array}{l}\text { Ativo não } \\
\text { corrente }\end{array}$ & $\begin{array}{c}\text { Ativo } \\
\text { corrente }\end{array}$ & $\begin{array}{c}\text { Passivo não } \\
\text { corrente }\end{array}$ & $\begin{array}{l}\text { Passivo } \\
\text { corrente }\end{array}$ & $\begin{array}{l}\text { Capital } \\
\text { próprio }\end{array}$ & $\begin{array}{l}\text { Média do } \\
\text { impacto }\end{array}$ \\
\hline \multirow[t]{2}{*}{ Dimensão } & 1 & 4,6 & 4,0 & 3,6 & 3,6 & 4,2 & 4.0 \\
\hline & 2 & 5,3 & 4,9 & 3,9 & 4,4 & 5,6 & 4.8 \\
\hline \multicolumn{2}{|c|}{$\begin{array}{l}\text { U de Mann- } \\
\text { Whitney }\end{array}$} & n.s. & 0,073 & n. s. & 0,079 & 0,022 & 0,032 \\
\hline \multirow{2}{*}{$\begin{array}{c}\text { Tempo de } \\
\text { serviço }\end{array}$} & 1 & 4,8 & 5,5 & 5,0 & 5,0 & 5,5 & 4.6 \\
\hline & 2 & 5,5 & 4,3 & 3,8 & 3,3 & 5,7 & 3.2 \\
\hline \multicolumn{2}{|c|}{$\begin{array}{c}\text { U de Mann- } \\
\text { Whitney ( } p \text { - value) }\end{array}$} & 0,045 & 0,074 & 0,004 & n.s. & 0,015 & 0.015 \\
\hline
\end{tabular}

Quanto à variável dimensão, constata-se que nas entidades de maior dimensão a expetativa é de um impacto superior (em termos médios) e que se fará sentir especialmente no capital próprio e no ativo não corrente.

Quanto ao tempo de serviço, refira-se que são os mais jovens que esperam maiores impactos em termos médios. Contudo, os funcionários com mais tempo de serviço apresentam expetativas superiores de variações no ativo não corrente e do capital próprio, mas, em termos médios, são mais moderados na sua apreciação.

\section{(4) Domínio técnico do SNC-AP.}

Quanto ao domínio técnico do novo normativo (inclui o conhecimento, a preparação, a dificuldade de adoção e o prestígio da profissão), a média das respostas situa-se próximo do valor quatro, com uma baixa dispersão nas respostas $(\overline{\mathrm{x}}=4,16, \sigma=0,892)$.

As respostas apresentam uma graduação em função das diversas fases de preparação, desde o conhecimento técnico das matérias até à sua implementação. Os inquiridos consideram que têm um conhecimento médio do SNC-AP $(\overline{\mathrm{x}}=4,2)$, mas revelam-se mal preparados $(\overline{\mathrm{x}}=3,9)$ o que cria dificuldades na sua implementação $(\overline{\mathrm{x}}=4,4)$. Interpreta-se estes resultados como a existência de condições técnicas para a transição de um normativo para o outro, mas com nível de insegurança significativo.

Tabela 6. Domínio técnico

\begin{tabular}{|c|c|c|c|c|c|c|c|c|c|}
\hline \multicolumn{2}{|c|}{$\begin{array}{c}\text { Conhecimento e } \\
\text { implementação }(\bar{x})\end{array}$} & \multicolumn{2}{|c|}{ Conhece } & \multicolumn{2}{c|}{ Preparado } & \multicolumn{2}{c|}{$\begin{array}{c}\text { Dificuldade de } \\
\text { adoção }\end{array}$} & \multicolumn{2}{c|}{$\begin{array}{c}\text { Prestígio } \\
\text { profissão }\end{array}$} \\
\hline Média & D p & Média & D p & Média & D p & Média & D p & Média & D p \\
\hline 4,16 & 0,811 & 4,2 & 1.117 & 3,9 & 1.296 & 4,4 & 1.775 & 4,8 & 1.600 \\
\hline
\end{tabular}

Considerando o género, refira-se que são as mulheres que declaram conhecer menos o SNC-AP $(\overline{\mathrm{x}}=3,6)$, com uma diferença significativa para os homens $(\overline{\mathrm{X}}=4,6)$. 
Tabela 7. Habilitações e domínio técnico

\begin{tabular}{|l|c|c|c|c|c|}
\hline \multicolumn{1}{|c|}{ Habilitações } & Conhece & Preparado & $\begin{array}{c}\text { Dificuldade } \\
\text { adoção }\end{array}$ & $\begin{array}{c}\text { Prestígio } \\
\text { profissão }\end{array}$ & $\begin{array}{c}\text { Conhecimento e } \\
\text { implementação }(\bar{x})\end{array}$ \\
\hline Licenciatura & 4,0 & 3,7 & 4,3 & 4,9 & 4,0 \\
\hline Pós graduações & 5,2 & 5,0 & 4,7 & 4,5 & 4,9 \\
\hline $\begin{array}{c}\text { U de Mann-Whitney } \\
\text { (p- value) }\end{array}$ & 0,016 & 0,023 & n.s. & n.s. & 0.04 \\
\hline
\end{tabular}

Nota: N.s. = não significativo $(p>0,05)$.

Considerando a antiguidade, são os que têm mais tempo de serviço que declaram conhecer menos o SNC-AP e em termos gerais são os que estão menos preparados, contudo declaram ter menos dificuldades na adoção do SNC-AP.

Também se verificam diferenças entre os profissionais certificados e não certificados quanto à preparação; estes últimos situam-se em médias substancialmente inferiores nos itens acima referidos, sendo a diferença significativa no item "Preparado para a transição" $(\overline{\mathrm{X}}=3,13)$ em relação aos profissionais certificados $(\overline{\mathrm{x}}=4,32)$. Também é nas unidades de maior dimensão que este item $(\overline{\mathrm{x}}=4,32, p$-value $=0,24)$ parece ser um fator de preocupação.

Tabela 8. Tempo de serviço e domínio técnico

\begin{tabular}{|c|c|c|c|c|c|}
\hline Tempo de serviço & Conhece & Preparado & $\begin{array}{c}\text { Dificuldade } \\
\text { adoção }\end{array}$ & $\begin{array}{c}\text { Prestígio } \\
\text { profissão }\end{array}$ & $\begin{array}{c}\text { Conhecimento e } \\
\text { implementação }(\bar{x})\end{array}$ \\
\hline Até 20 anos & 4,5 & 4,0 & 4,7 & 4,9 & 4,40 \\
\hline Mais de 20 anos & 3,5 & 3,6 & 3,5 & 4,6 & 3,53 \\
\hline $\begin{array}{c}\text { U de Mann- } \\
\text { Whitney (p- value) }\end{array}$ & 0,045 & n.s. & n.s. & n.s. & 0.012 \\
\hline
\end{tabular}

Nota: N.s. = não significativo $(p>0,05)$.

\section{Conclusões}

Face às alterações registadas nas últimas décadas ao nível económico-financeiro, em geral, e à evolução das economias periféricas da União Europeia, em particular, a questão do modelo de contabilidade a ser aplicado às entidades do setor público, nomeadamente às entidades do SNS, tem vindo a ganhar uma crescente relevância. Nomeadamente, enquanto mecanismo que proporcione melhorias na qualidade e na transparência do relato financeiro, mormente ao facultar informação para uma gestão financeira de qualidade na tomada de decisão. O processo de implementação do SNC-AP envolve uma pluralidade de sujeitos, entre outros, o poder político, os reguladores, os dirigentes e os responsáveis pela contabilidade dos organismos públicos. Não é irrelevante, para as conclusões deste estudo, referir que para as entidades do SNS, concretamente as Entidades Públicas Empresariais, apesar de contabilizarem em POC-MS, divulgam em SNC para a tutela, facto que terá incentivado os responsáveis pela contabilidade destas entidades a uma atualização técnica no âmbito deste enquadramento contabilístico, diminuindo, desta forma, o seu défice de conhecimento em relação ao SNC-AP.

O estudo sobre a opinião dos responsáveis da contabilidade das trinta e seis entidades do SNS permitiu concluir que, em geral, estes profissionais acolhem de forma positiva esta 
alteração e esperam que as futuras DF sejam mais úteis para os utilizadores, apesar de reconhecerem dificuldades técnicas na transição. A valorização semelhante atribuída quer ao papel do POC-MS, quer ao do SNC-AP, não permite concluir de forma inequívoca que estes profissionais sejam de opinião que haja a necessidade de substituição urgente do atual normativo contabilístico. Mais ainda, estes profissionais confrontam a sua capacidade de dar resposta ao cumprimento da legalidade, reconhecendo, contudo, uma maior qualidade das DF e a sua utilidade para os utilizadores, opinião que vai ao encontro dos objetivos subjacentes à publicação do SNC-AP, no que concerne ao incremento dos aspetos qualitativos da informação financeira. Também reconhecem que o prestígio da profissão pode ser reforçado em associação com o SNC-AP.

Identifica-se uma posição de defesa do POC-MS por parte dos profissionais com mais tempo de serviço, que não anteveem melhorias significativas com a mudança de normativo. Consideram-se estes resultados consistentes com os estudos que incidem sobre a resistência à mudança que é mais intensa naqueles que já trabalham há mais tempo e que consequentemente têm mais idade (vide Lima, Carrieri \& Pimentel, 2007).

A questão da comparabilidade das DF seja em sede do POC-MS, seja no âmbito do novo normativo, não parece ser um aspeto relevante, contrariando um dos argumentos principais da utilização de normas harmonizadas num contexto internacional.

Estes profissionais esperam impactos patrimoniais (balanço) maioritariamente negativos, quer nas rubricas dos ativos, quer na dos passivos não correntes, contudo com um resultado final positivo nos capitais próprios. Os resultados sugerem que o impacto nas contas de balanço é uma preocupação maior nas entidades de maior dimensão.

É reconhecida alguma preocupação com a transição para o novo normativo, principalmente em alguns grupos específicos onde os mais habilitados anteveem maiores dificuldades, enquanto os profissionais com mais anos de serviço e os profissionais não certificados anteveem menos dificuldades, apesar de se sentirem menos preparados tecnicamente.

No que se refere às diferenças encontradas na variável género avança-se como explicação a defendida por Santos e Amâncio (2014), que demonstram que em situações de minoria (a amostra é $61 \%$ masculina), em profissões tradicionalmente masculinas, as mulheres são menos proativas.

Em relação ao estudo de Nunes et al. (2016) releva-se especialmente a alteração da preferência pelo SNC-AP, deduzida da análise das opiniões dos novos elementos da amostra (contabilistas não certificados) que, em regra, exprimem maior desconforto e desconhecimento em relação ao novo normativo. Avança-se como explicação o facto dos contabilistas certificados, por exigência da Ordem dos Contabilistas Certificados, estarem obrigados à frequência de ações de formação, pelo que lhes permitiu a aquisição dos conhecimentos necessários. Os restantes resultados vão na linha dos obtidos no estudo acima referido.

Como limitação deste estudo é de destacar a baixa taxa de respostas dos preparadores que não têm a qualificação de contabilista certificado, o que pode contribuir para algum enviesamento dos resultados.

Porém, este estudo representa um contributo importante para a investigação na medida em que afere a opinião dos responsáveis pela contabilidade do SNS relativamente à introdução do SNC-AP e antecipa os impactos da adoção do mesmo nos profissionais que 
serão chamados para a sua implementação, permitindo que entidades como a Administração Central do Sistema de Saúde desenvolvam os mecanismos necessários para apoiar a implementação deste processo.

A verdade é que as associações profissionais de contabilistas e auditores e outros stakeholders associados podem exercer grandes pressões sobre as mudanças contabilísticas, uma vez que a adoção do SNC-AP “vai ajudá-los a conquistar novos clientes e a conseguir um aumento do volume de negócios” (Oulasvirta, 2014: 275).

Por fim, é de referir que se considera oportuno o acompanhamento do processo de transição para o SNC-AP, com o propósito de perceber as consequências que a sua adoção terá efetivamente tido em termos de qualidade e comparabilidade das DF (recorde-se que estes são objetivos cimeiros da introdução do novo normativo). Para além disso, seria pertinente alargar a investigação aos Municípios que aplicam o Plano Oficial de Contabilidade das Autarquias Locais (POCAL), de modo que se espera que estudos futuros possam seguir esta linha de investigação.

\section{Referências bibliográficas}

Barbosa, J. (2009). A harmonização contábil das NICSP na contabilidade pública: O caso do Brasil e Portugal (Dissertação de mestrado não publicada). Escola de Economia e Gestão, Universidade do Minho, Braga.

Coelho, J. (2014). O sistema de informação financeira no ensino secundário público em Portugal. (Tese de doutoramento não publicada). Universidade de Lisboa, Lisboa.

Correia, C. \& Gonçalves, M. (2010). Activos fixos tangíveis na contabilidade pública e empresarial- IPSS 17 versus IAS 16. Revista de Contabilidade \& Empresas, 6 (2), 19-24.

Cruz, A. (2012). Contributo para um novo sistema de normalização contabilística para o setor público em Portugal (Dissertação de mestrado não publicada). ISCTE, Lisboa.

Decreto-Lei n. ${ }^{\circ} 232 / 1997$, de 3 de setembro do Ministério das Finanças. Diário da República I Série- A n. ${ }^{\circ}$ $203,4594-4638$.

Decreto-Lei n. ${ }^{\circ}$ 192/2015, de 11 de setembro do Ministério das Finanças. Diário da República I Série n. ${ }^{\circ}$ $178,7584-7828$.

Ferreira, C. (2013). Harmonização contabilística no setor público: Constrangimentos na adoção das IPSAS (Dissertação de mestrado não publicada). Instituto Superior de Ciências Sociais e Políticas, Universidade de Lisboa, Lisboa.

França, A. \& Jesus, M (2014, setembro). Impacto das IPSAS na investigação em contabilidade pública nos últimos cinco anos. Comunicação apresentada no XVI congresso AECA, Leiria.

Gomes, P., Fernandes, M. \& Carvalho, J. (2015). O processo de harmonização internacional da contabilidade pública em Portugal: A perspetiva dos diferentes stakeholders. Comunicação apresentada no $V$ Congresso dos TOC, Lisboa.

Gonçalves, C. (2011). A accountability nos serviços e fundos autónomos (Dissertação de mestrado não publicada). Escola Superior de Contabilidade e Administração de Lisboa, Instituto Politécnico de Lisboa, Lisboa.

Jorge, S. (2012a). Novas tendências da contabilidade pública: Portugal numa perspectiva internacional (I). Revista da OTOC, 152, 47-52.

Jorge, S. (2012b). Novas tendências da contabilidade pública: Portugal numa perspectiva internacional (II). Revista da OTOC, 153, 40-45.

Lima, M., Carrieri, A. \& Pimentel, T. (2007). Resistência à mudança gerada pela implementação de sistemas de gestão integrada (ERP): Um estudo de caso. Revista Gestão e Planejamento, 8 (1), 89105.

Marconi, M. \& Lakatos, E. (1999). Técnicas de Pesquisa (4. ${ }^{a}$ edição). São Paulo: Atlas.

Nogueira, S. \& Carvalho, J. (2006). A contabilidade pública em Portugal: Opinião de especialistas. Comunicação apresentada no XIII Encuentro AECA, Córdoba.

Nunes, C., Fernandes, J. \& Gonçalves, C. (2016). A perceção dos contabilistas certificados das Unidades Hospitalares na adoção do SNC-AP. Revista Portuguesa de Contabilidade, 23 (6), 446-456. 
Oulasvirta, L. (2014). The reluctance of a developed country to choose international public sector accounting standards of the IFAC: a critical case study. Critical Perspectives on Accounting, 25 (3), 272-285. http://dx.doi.org/10.1016/j.cpa.2012.12.001

Pinho, S. (2014). O Impacto da adoção das IPSAS nas universidades portuguesas (Dissertação de mestrado não publicada). Instituto Superior de Contabilidade e Administração de Aveiro, Universidade de Aveiro, Aveiro.

Pires, A. (2009). Sistema de normalização contabilística - do POC ao SNC. Lisboa: Publisher Team.

Rodrigues, C. (2015). A adoção das IPSAS pela primeira vez no setor público português: Estudo de caso distrito de Lisboa (Dissertação de mestrado não publicada). Escola Superior de Ciências Empresariais, Instituto Politécnico de Setúbal, Setúbal.

Ryan, B., Scapens, R. \& Theobald, M. (2002). Research method and methodology in finance and accounting. (2. ${ }^{a}$ edição). Londres: Thomson.

Santos, M. e Amâncio, L. (2014). Sobreminorias em profissões marcadas pelo género: Consequências e reações. Análise Social, 212, xlix $\left(30^{\circ}\right)$.

Santos, P. \& Pinho C. (2014, setembro). A adopção das IPSAS em Portugal conduzirá necessariamente ao relato de informação financeira comparável no âmbito do Sector Público Administrativo? Comunicação apresentada no XVI congresso AECA, Leiria.

Silva, J. \& Carvalho, F. (2007). Estudo comparativo entre a IPSAS 19 e o Plano Oficial de Contabilidade Pública. Comunicação apresentada no XIV Encuentro AECA, Valência.

Agradecimentos

Este artigo é financiado por Fundos Nacionais através da FCT - Fundação para a Ciência e a Tecnologia no âmbito do projeto UID/SOC/04020/2013.

CARLOS NUNES é professor adjunto convidado da Escola Superior de Gestão, Hotelaria e Turismo da Universidade do Algarve, onde leciona unidades curriculares na área da contabilidade, em cursos de licenciatura e de especialização tecnológica. Mestre em Contabilidade pela Faculdade de Economia, Universidade do Algarve e detentor do Título de Especialista na área de Contabilidade e Fiscalidade (CNAEF 344). É Contabilista Certificado no Centro Hospitalar do Algarve EPE, pelo que tem lecionado diversas ações de formação dirigidas a Contabilistas Certificados. Endereço institucional: Campus da Penha, Estrada da Penha, 8005-139 Faro, Portugal.

JOAQUIM SANT'ANA FERNANDES é professor adjunto da Escola Superior de Gestão, Hotelaria e Turismo da UALG onde leciona unidades curriculares na área da contabilidade, em cursos de licenciatura e mestrado. Doutor em Administração de empresas. É coautor dos livros Relato Financeiro: Interpretação e Análise e Contabilidade Financeira Explicada. Leciona em diversas ações de formação dirigidas a profissionais de contabilidade e tem coordenado diversos estudos económicos. É Investigador associado do Centro de Investigação sobre o Espaço e as Organizações (CIEO), Endereço institucional: Campus da Penha, Estrada da Penha, 8005-139 Faro, Portugal.

CRISTINA GONÇALVES é professora adjunta da Escola Superior de Gestão, Hotelaria e Turismo da Universidade do Algarve onde leciona desde 1992. Mestre em Ciências Empresariais. Tem ministrado diversas disciplinas na área da Contabilidade, em curso de licenciatura e de mestrado. É coautora de diversos livros sobre contabilidade e ética profissional. É convidada regularmente para ministrar cursos de formação. É investigadora associada do Centro de Investigação sobre o Espaço e as Organizações (CIEO). Endereço institucional: Campus da Penha, Estrada da Penha, 8005-139 Faro, Portugal.

Submetido em 26 outubro 2016

Aceite em 4 janeiro 2017 
Apêndice A. Lista das entidades pertencentes ao Serviço Nacional de Saúde (SNS)

\begin{tabular}{|c|}
\hline $\begin{array}{l}\text { Entidades SPA } \\
\end{array}$ \\
\hline ADMINISTRAÇÃO CENTRAL DO SISTEMA DE SAÚDE, IP \\
\hline INSTITUTO PORTUGUÊS DO SANGUE E DA TRANSPLANTAÇAO, IP \\
\hline INSTITUTO NACIONAL DE SAÚDE DR. RICARDO JORGE, IP \\
\hline HOSPITAL DR. FRANCISCO ZAGALO, IP \\
\hline HOSPITAL ARCEBISPO JOÃO CRISÓSTOMO, IP \\
\hline CENTRO MÉDICO DE REABIL. DA REG. CENTRO - ROVISCO PAIS, IP \\
\hline INSTITUTO OFTALMOLÓGICO DR. GAMA PINTO, IP \\
\hline ADMINISTRAÇÃO REGIONAL DE SAÚDE DO NORTE, IP \\
\hline ADMINISTRAÇÃO REGIONAL DE SAÚDE DO CENTRO, IP \\
\hline ADMINISTRAÇÃO REGIONAL DE SAÚDE DE LISBOA E VALE DO TEJO, IP \\
\hline ADMINISTRAÇÃO REGIONAL DE SAÚDE DO ALENTEJO,IP \\
\hline ADMINISTRAÇÃO REGIONAL DE SAÚDE DO ALGARVE, IP \\
\hline CENTRO HOSPITALAR PSIQUIÁTRICO DE LISBOA, IP \\
\hline CENTRO HOSPITALAR DO OESTE, IP \\
\hline Entidades EPE \\
\hline CENTRO HOSPITALAR COVA DA BEIRA, EPE \\
\hline CENTRO HOSPITALAR MÉDIO TEJO, EPE \\
\hline HOSPITAL DISTRITAL FIGUEIRA DA FOZ, EPE \\
\hline HOSPITAL DISTRITAL S. MARIA MAIOR, EPE - BARCELOS \\
\hline HOSPITAL DISTRITAL SANTARÉM, EPE \\
\hline HOSPITAL GARCIA DE ORTA, EPE \\
\hline UNIDADE LOCAL DE SAÚDE DE MATOSINHOS, EPE \\
\hline INSTITUTO PORTUGUÊS DE ONCOLOGIA DE COIMBRA, EPE \\
\hline INSTITUTO PORTUGUÊS DE ONCOLOGIA DE LISBOA, EPE \\
\hline INSTITUTO PORTUGUÊS DE ONCOLOGIA DO PORTO, EPE \\
\hline CENTRO HOSPITALAR DE LISBOA - ZONA OCIDENTAL, EPE \\
\hline CENTRO HOSPITALAR DE SETUBAL, EPE \\
\hline HOSPITAL ESPÍRITO SANTO DE ÉVORA, EPE \\
\hline CENTRO HOSPITALAR DE LISBOA CENTRAL, EPE \\
\hline CENTRO HOSPITALAR TRÁS-OS-MONTES E ALTO DOURO, EPE \\
\hline CENTRO HOSPITALAR MÉDIO AVE, EPE \\
\hline CENTRO HOSPITALAR VILA NOVA GAIA/ESPINHO, EPE \\
\hline UNIDADE LOCAL SAÚDE NORTE ALENTEJANO, EPE \\
\hline CENTRO HOSPITALAR DO PORTO, EPE \\
\hline CENTRO HOSPITALAR DO TÂMEGA E SOUSA, EPE \\
\hline CENTRO HOSPITALAR LISBOA NORTE, EPE \\
\hline CENTRO HOSPITALAR PÓVOA VARZIM / VILA DO CONDE, EPE \\
\hline UNIDADE LOCAL DE SAÚDE ALTO MINHO, EPE \\
\hline UNIDADE LOCAL DE SAÚDE DA GUARDA, EPE \\
\hline UNIDADE LOCAL DO BAIXO ALENTEJO, EPE \\
\hline HOSPITAL DE MAGALHÃES LEMOS, EPE \\
\hline CENTRO HOSPITALAR DE ENTRE O DOURO E VOUGA, EPE \\
\hline HOSPITAL FERNANDO DA FONSECA, EPE \\
\hline CENTRO HOSPITALAR DO BARREIRO - MONTIJO, EPE \\
\hline UNIDADE LOCAL DE SAÚDE DE CASTELO BRANCO, EPE \\
\hline CENTRO HOSPITALAR DE SÃO JOÃO, EPE \\
\hline CENTRO HOSPITALAR E UNIVERSITÁRIO DE COIMBRA, EPE \\
\hline CENTRO HOSPITALAR DO BAIXO VOUGA, EPE \\
\hline CENTRO HOSPITALAR TONDELA-VISEU, EPE \\
\hline CENTRO HOSPITALAR DE LEIRIA, EPE \\
\hline UNIDADE LOCAL DE SAÚDE DO NORDESTE, EPE \\
\hline UNIDADE LOCAL DE SAÚDE DO LITORAL ALENTEJANO, EPE \\
\hline CENTRO HOSPITALAR DO ALGARVE, EPE \\
\hline CENTRO HOSPITALAR ALTO AVE, EPE \\
\hline
\end{tabular}

Fonte: informação disponível em www.acss.min-saude.pt/ 